\title{
Everyday flexibility and functional milestones in anorexia nervosa: survey results from a mixed community sample
}

\author{
Kelly M. Dann ${ }^{1}$. $\cdot$ Phillipa $\mathrm{Hay}^{2} \cdot$ Stephen Touyz ${ }^{3}$
}

Received: 7 July 2021 / Accepted: 8 September 2021 / Published online: 22 September 2021

(c) The Author(s), under exclusive licence to Springer Nature Switzerland AG 2021

\begin{abstract}
Purpose This study examined the relationship between self-reported cognitive-behavioral flexibility scores on the Eating Disorder Flexibility Index (EDFLIX) and objective social and occupational functional milestones in participants with a lifetime diagnosis of anorexia nervosa (AN). The Work and Social Adjustment Scale (WSAS) was included to compare objective and subjective measures.

Methods 114 female adult participants with a current (53.5\%) or past (46.5\%) full or partial AN syndrome diagnosis completed an online survey which included functional milestone questions, the EDFLIX, WSAS, EDE-Q, and DASS-21.

Results Everyday flexibility scores were significantly associated with WSAS scores, but not functional milestones for the same domain. Lower flexibility was related to higher WSAS work impairment but was not associated with poor occupational outcomes. Lower flexibility was related to higher WSAS social impairment but was not associated with less frequent social contact with friends. Milestones across work, social and relationship areas were not significantly correlated, suggesting individuals have areas of strength and weakness across functional domains. In contrast, WSAS ratings indicated broad functional impairment.

Conclusion Results from the milestones suggest self-reported cognitive-behavioral flexibility is not a strong determinant of everyday function. Results from the subjective WSAS function measure and the more objective functional milestones were not consistent. To obtain a more balanced assessment of everyday functioning in AN, both subjective and objective measures should be considered.

Level of evidence Level III Case-control analytic study.
\end{abstract}

Keywords Anorexia nervosa $\cdot$ Cognitive-behavioral flexibility $\cdot$ Functional outcome $\cdot$ Work $\cdot$ Social function $\cdot$ Eating disorders

\section{Introduction}

A consistent finding in the literature is that individuals with Anorexia Nervosa (AN) perform more poorly on neuropsychological tests of cognitive flexibility than healthy control groups [45]. Cognitive flexibility is the ability to rapidly shift focus between mental sets or tasks in response

Kelly M. Dann

kelly.dann@sydney.edu.au

1 School of Psychology, The University of Sydney, Sydney, NSW 2006, Australia

2 School of Medicine, Western Sydney University, Sydney, Australia

3 InsideOut Institute and School of Psychology, The University of Sydney, Sydney, Australia to changing demands $[11,27]$. In line with clinical observations of cognitive-behavioral rigidity in individuals with $\mathrm{AN}$, poor cognitive flexibility is considered a key risk factor in the etiology and maintenance of the disorder [34, 40]. Individuals with AN also experience difficulty in their dayto-day function. Social and occupational function has been reported to be severely impaired, with greater impairment associated with lower BMI and more severe eating disorder symptoms [18, 39, 41].

In the wider neuropsychiatric literature, there has been a specific emphasis on whether poor performance on neurocognitive tests is associated with functional impairments. Results in schizophrenia suggest a substantial general effect of cognitive issues on overall functional outcome, with a greater impact on occupational than social function [17], and general effects are also found in bipolar [10], and 
major depressive disorders [13]. In AN, despite increasing interest in associations between cognitive flexibility and clinical characteristics, little empirical work has been undertaken to assess the possible impact on everyday function. The available research suggests cognitive flexibility measured using standard neuropsychological performance tests is not strongly associated with functional outcome in AN [9]. However, self-report measures of cognitivebehavioral flexibility appear to be sensitive to the everyday issues experienced by adolescents with $\mathrm{AN}$, and indicate that behavioral response shifting may be more problematic than cognitive shifting [25, 42].

Although cognitive flexibility is usually measured in AN with performance tests such as the Wisconsin Card Sort Test [16], self-reported flexibility scales have also been developed to improve the ecological validity of measurement by capturing the behavioral manifestations of cognitive issues as they occur in daily life. Disorderspecific scales include the Detail and Flexibility Questionnaire (DFlex; [33]), which assesses cognitive rigidity and attention to detail in daily life, and the Eating Disorder Flexibility Index (EDFLIX; [8]), which measures general and eating disorder-specific everyday flexibility. Although performance tests and self-report measures of cognitive flexibility appear to capture different aspects of the same construct [22], results in adolescent AN cohorts have demonstrated the utility of self-report data in observing associations between flexibility issues and functional outcomes which have proven difficult to identify with performance tests.

Functional outcome in AN is usually assessed with selfreport measures such as the Clinical Impairment Assessment (CIA; [3]), or the Work and Social Adjustment Scale (WSAS; [29]). The CIA has been used to compare performance measures of cognitive flexibility with functional outcome, however, significant associations have not been noted (Oldershaw, Lavender, \& Schmidt, 2018; [32]. These results are consistent with a broad finding that associations between cognitive performance tests and self-reported functional outcome are also generally low [23, 36]. However, better cognitive flexibility as measured by the Brixton Test [5] has recently been associated with lower ratings for functional impairment as measured by the WSAS [12].

Although self-report measures of cognitive-behavioral flexibility have demonstrated utility, comparing self-reported flexibility and self-reported function is methodologically problematic as associations may be inflated by overlap in the response style or self-perception of the reporter, or overlap in the constructs assessed, which in extreme cases may include overlap in the wording of items. And although performance function tests are available, most are designed for cohorts with cognitive issues of a general rather than specific nature.
An alternative approach to measuring real-life performance is the attainment and maintenance of functional milestones. Milestones such as being currently employed or living independently provide an objective measure of functional strengths and weaknesses, which may be particularly important in cohorts with high performance expectations. Maintaining employment or studying for a higher degree also requires high levels of sustained efficiency, and may therefore be more sensitive to specific rather than general cognitive issues [31].

Functional milestones have provided useful data regarding the effects of cognitive variables on outcomes in other psychiatric disorders. In schizophrenia, correlations between functional milestones across different areas of everyday function are reported to be very low [19], and to be impacted by different cognitive deficits [37]. In Obsessive-Compulsive Disorder, a specific relationship between flexibility and function has been noted; poor performance on the Trail Making Test (TMT-B) was significantly associated with poorer vocational outcomes [31].

The specificity of the impact of cognitive issues on functional outcome demonstrated in other psychiatric disorders suggest an individual may experience effects in one area, yet perform normally, or even very well, in another. This is consistent with the observation that individuals with AN may be high achievers in some areas of their lives whilst facing struggles in others. This has not been a focus of research in $\mathrm{AN}$, but there is some empirical support for the suggestion that everyday function in AN may not be impaired in all areas, even with a chronic course of illness [2]. Self-reported quality of life has also been noted to vary across areas of function in AN [38].

\section{Current study}

The aim of the current study was to investigate the relationship between cognitive-behavioral flexibility and everyday function in an adult community sample of individuals with a lifetime diagnosis of AN. Previous research suggests cognitive flexibility measured using performance tests is not strongly associated with self-reported functional outcome in AN. Therefore, the current research measures self-reported cognitive-behavioral flexibility and objective social and occupational functional outcomes. To provide a comparison with past research in AN, we also included the Work and Social Adjustment Scale. We aimed to address the following research questions a. What is the relationship between functional milestones across different areas of everyday function (social, occupational) in AN? b. Is self-reported cognitivebehavioral flexibility associated with different areas of function? and c. Is the pattern of results different for participants with a current versus a former diagnosis? It was predicted that participants who reported better flexibility would have 
Table 1 Sample demographic and clinical characteristics

\begin{tabular}{lll}
\hline & $N$ & $\%$ \\
\hline Ethnicity & & \\
Caucasian & 101 & $88.6 \%$ \\
Asian & 4 & $3.5 \%$ \\
Mixed Ethnicity (Asian/ Caucasian) & 2 & $1.75 \%$ \\
Indigenous Australian & 2 & $1.75 \%$ \\
Other & 5 & $4.4 \%$ \\
Diagnosis & & \\
Current & 61 & $53.5 \%$ \\
Past & 53 & $46.5 \%$ \\
AN Diagnostic subtype & & \\
AN-R & 71 & $62.3 \%$ \\
AN-BP & 23 & $20.2 \%$ \\
OSFED/ Atypical AN & 7 & $6.1 \%$ \\
EDNOS-AN & 5 & $4.4 \%$ \\
Unknown & 8 & $7 \%$ \\
Highest level of education & & \\
University Postgraduate & 30 & $26.3 \%$ \\
University Undergraduate & 42 & $36.8 \%$ \\
Tertiary certificate, TAFE & 15 & $13.2 \%$ \\
Higher school certificate (Yr. 12) & 23 & $20.2 \%$ \\
School certificate (Yr. 10) & 4 & $3.5 \%$ \\
\hline
\end{tabular}

better functional outcomes, and that participants with a current diagnosis would report more difficulties with both flexibility and function than participants with a past diagnosis.

\section{Method}

\section{Participants and procedure}

Participants were 114 female adults $\left(17^{1}-57\right.$ years, $\left.M=28.6\right)$ who reported having a current $(53.5 \%)$ or past $(46.5 \%)$ diagnosis of AN. The majority of participants identified as Caucasian and were born in Australia. See Table 1 for the full demographic statistics of the sample. Participants were required to be aged over 18 years and have a current or past formal diagnosis of AN from a medical professional. No exclusion criteria were applied, including gender, however, all respondents were female. Participants who reported a partial AN syndrome diagnosis (EDNOS-AN, OSFED-AN, Atypical AN) were included in the sample. Participants were recruited through advertisements placed on research pages of university and eating disorder organization websites, and their associated newsletters. Participants completed

${ }^{1}$ One participant was slightly under the eligibility requirement of 18 years. the study as an online questionnaire after providing their informed consent. Data was collected from May to November 2020, and the study was approved by The University of Sydney Human Research Ethics Committee, approval number 2020/176.

\section{Measures}

Demographic and clinical variables. Participants reported their age, country of birth, ethnicity, whether they had a current or past diagnosis, diagnostic subtype, the approximate total duration of their disorder, and whether they had been admitted for in-patient treatment.

The Eating Disorder Flexibility Index (EDFLIX; [8]) is a 36-item self-report scale assessing general and ED-specific flexibility in everyday life. Subscales for General Flexibility, Food and Exercise Flexibility and Weight and Shape Flexibility can be calculated. Total scores range from 36 to 216 , with higher scores indicating greater flexibility. Cronbach's alpha reported in a mixed ED sample in the validation study was 0.91 [8], and in this study was 0.95 .

The Work and Social Adjustment Scale (WSAS; [29]) is a brief 5-item validated self-report measure of the degree of disorder-related impairment in five areas of functioning: ability to work, home management, social leisure, private leisure and the ability to maintain close relationships. Ratings are made on an 8-point scale (Not at all, Slightly, Definitely, Markedly, Very Severely). Total scores range from 0 to 40 , with scores over 20 indicting clinically significant impairment. Cronbach's alpha between 0.81 and 0.94 have been reported for participants with depression and 0.79 and 0.88 in OCD [29]. Cronbach's alpha in this study was 0.90 .

Functional milestones for occupational and relationship outcome were based on those used in OCD by Perna et al. [31]. Dichotomous occupational outcome was defined as being employed (full or part-time) or being a current student with adequate performance vs. being unemployed (regardless of duration), or being a student not demonstrating adequate performance (classes failed/incomplete). Dichotomous relationship outcome was defined as: Being married or in a long-term relationship (current or former), versus never having been in a long-term relationship.

Social function was further assessed by two additional sets of questions. As an objective measure of social leisure function, participants were asked to report the frequency of social contact (face-to-face meetings) with friends; ratings were made on a 4-point scale (daily, weekly, monthly, rarely). Because the dichotomous long-term relationship question may not capture variation in younger adult participants, we also asked participants to rate their relationship over the past six months with their parents, siblings, partner and children. Ratings were made on a 4-point scale 
(no contact, not good, good, very good or not applicable). Ratings from these sets of questions were used to calculate a composite score for social contact with friends, and quality of close interpersonal relationships, based on the procedure used by Holst and Thorell [21]. Since responses were collected during a period where participants may have been affected by COVID-19 restrictions, participants were asked to reply to all questions based on what was normal for them prior to any restrictions.

The Eating Disorder Examination Questionnaire (EDE$\mathrm{Q}$; [14] is a 28-item self-report questionnaire validated with Australian norms [28] used to assess eating disorder attitudes and behaviors over the past 28 days. Items assessing ED-related attitudes comprise a global score with a range from 0 to 6 . Higher scores indicate more severe eating disorder pathology. The remaining items assess the frequency of ED behaviors: objective and subjective bingeing, purging, laxative use and compulsive exercise. Height and weight are reported, and BMI $\left(\mathrm{kg} / \mathrm{m}^{2}\right)$ was calculated from this data. Cronbach's alpha of 0.93 has been reported in an Australian female community sample [28], and in this study was 0.96 .

The Depression Anxiety Stress Scale short form (DASS21; [24] is a 21-item questionnaire which assesses mood symptoms over the past week. Items are rated on a 4-point scale from $0=$ Never to $3=$ Almost always, and scales for depression, anxiety and stress are calculated. Higher scores indicate greater psychopathology. Cronbach's alpha of the depression, anxiety and stress scales in a general population sample have been reported as $0.88,0.82$ and 0.90 respectively [20], and in this study were $0.94,0.90$ and 0.89 .

\section{Statistical analyses}

Data analysis was conducted using IBM SPSS Statistics 24.0 software. To control for the impact of testing multiple dependent measures, tests were run with an alpha level of 0.01 . Effects that reached the conventional 0.05 criterion are noted as marginal. Relationships between each of the studied variables were assessed using Spearman rank-order correlation coefficient $\left(r_{\mathrm{s}}\right)$, interpreted as $<0.19=$ very weak, $0.20-0.39=$ weak, $0.40-0.59=$ moderate, 0.60 $0.79=$ strong,$>0.79=$ very strong. Where correlations indicated a significant association, a linear or logistic regression was conducted to examine whether cognitive-behavioral flexibility scores remained significantly associated with functional outcomes beyond known covariates. Covariates were depression, anxiety and stress scores based on past AN literature [1], age to control for possible age bias in the long-term relationship milestone, BMI to control for possible impact on work participation, and EDE-Q scores based on strong associations in our initial correlation analysis. An a priori power analysis conducted using G-Power [15] based on the effect size for TMT-B on occupational outcome in
OCD [31] indicated our analysis required a minimum sample size of 86 participants given an alpha of 0.01 at $80 \%$ power. Group differences between participants with current versus former diagnoses were assessed with one-way ANOVA and Pearson chi-square. Differences in strength of cognitivebehavioral flexibility scores on functional outcomes between participants with current and former diagnoses was analyzed by the introduction of a flexibility $\mathrm{x}$ group interaction term in the regression analyses.

\section{Results}

\section{Demographic and clinical characteristics}

See Table 1 for descriptive statistics of the study sample. There was no significant difference in age, attainment of the relationship milestone, or in frequency of social contact with friends between participants with a current versus a past diagnosis. However, participants with a past diagnosis reported a shorter duration of illness, were less likely to have been admitted for in-patient treatment, had higher flexibility scores, reported lower WSAS work, social and relationship impairment, were more likely to attain the occupational milestone, provided higher ratings for quality of close interpersonal relationships, had lower DASS-21 depression, anxiety and stress scores, and higher BMI. Difference statistics are displayed in Table 2. EDE-Q global scores for participants with a current diagnosis were in the 95th percentile, and the 75th percentile for those with a past diagnosis [28]. Mean DASS-21 scores for participants with a current diagnosis were in the severe range, and the moderate range for those with a past diagnosis. Although flexibility scores were higher for participants with a past diagnosis, mean EDFLIX scores were within the clinical range $(<136)$ for both groups.

\section{Correlation analysis}

\section{Correlations between measures of work and social function}

Bivariate correlations are available in Supplementary Materials. WSAS scores for impairment on occupational, social leisure and interpersonal function showed moderate to strong correlations $\left(r_{\mathrm{s}}=0.58-0.69, p<0.01\right)$. Dichotomous occupational and social milestones and extended social function composite scores for social contact with friends and quality of close interpersonal relationships were not significantly correlated $\left(r_{\mathrm{s}}<0.17\right)$. Dichotomous milestones and social function composite scores were negatively associated with WSAS scores for the same domains; lower impairment was 
Table 2 Mean (SD) demographics and scores with group comparisons

\begin{tabular}{|c|c|c|c|c|c|c|}
\hline & \multicolumn{2}{|c|}{ Current diagnosis } & \multicolumn{2}{|c|}{ Past diagnosis } & \multirow[t]{2}{*}{ Test statistic } & \multirow[t]{2}{*}{$p$} \\
\hline & $N$ & $M(S D)$ or $\%$ & $N$ & $M(S D)$ or $\%$ & & \\
\hline Age (years) & 61 & $28.0(8.8)$ & 53 & $29.2(8.9)$ & $F(1,112)=.48$ & 0.49 \\
\hline Duration of illness (years) & 61 & $11.2(9)$ & 53 & $7.4(6.2)$ & $F(1,112)=6.8$ & 0.01 \\
\hline Have received inpatient treatment & 42 & $68.9 \%$ & 23 & $43.4 \%$ & $X^{2}(1)=7.5$ & 0.006 \\
\hline BMI & 58 & $19.0(4.1)$ & 43 & $21.8(2.8)$ & $F(1,99)=15.2$ & $<0.001$ \\
\hline EDFLIX total score & 58 & $90.8(22.8)$ & 47 & $123.6(32.6)$ & $F(1,103)=36.5$ & $<0.0001$ \\
\hline Milestone: work (attained) & 45 & $73.8 \%$ & 50 & $94.3 \%$ & $X^{2}(1)=8.6$ & 0.003 \\
\hline Milestone: relationship (attained) & 25 & $41 \%$ & 33 & $62.3 \%$ & $X^{2}(1)=5.1$ & 0.02 \\
\hline Social contact with friends & 61 & $5.5(1.8)$ & 53 & $5.7(1.6)$ & $F(1,112)=.47$ & 0.50 \\
\hline Quality of close relationships & 61 & $1.9(.6)$ & 53 & $2.3(.6)$ & $F(1,112)=9.9$ & 0.002 \\
\hline WSAS work & 61 & $4.1(2.3)$ & 51 & $1.9(2.2)$ & $F(1,110)=28.7$ & $<0.001$ \\
\hline WSAS social & 61 & $6.3(1.6)$ & 51 & $3.4(2.7)$ & $F(1,112)=49.2$ & $<0.001$ \\
\hline WSAS relationship & 61 & $5.3(2.2)$ & 51 & $3.2(2.7)$ & $F(1,112)=18.3$ & $<.001$ \\
\hline DASS depression & 58 & $26.3(11.4)$ & 49 & $14.3(12.6)$ & $F(1,105)=27.0$ & $<0.001$ \\
\hline DASS anxiety & 58 & $18.7(10.6)$ & 49 & $12.2(11.5)$ & $F(1,105)=9.0$ & 0.003 \\
\hline DASS stress & 58 & $27.5(8.7)$ & 49 & $18.8(10.5)$ & $F(1,105)=21.7$ & $<0.001$ \\
\hline EDE-Q global score & 58 & $4.3(1.3)$ & 44 & $2.5(1.8)$ & $F(1,100)=35.1$ & $<0.001$ \\
\hline
\end{tabular}

Significant p-values in bold

$B M I$ Body Mass Index, DASS Depression Anxiety Stress Scale short form, EDE- $Q$ Eating Disorder

Examination Questionnaire, EDFLIX Eating Disorder Flexibility Index, WSAS Work and Social Adjustment Scale related to attainment of the milestones, more social contact and higher ratings for the quality of interpersonal relationships $\left(r_{\mathrm{s}}=-0.27\right.$ to $\left.-0.40, p<0.01\right)$.

\section{Correlations between cognitive-behavioral flexibility and work and social function}

Lower scores for cognitive-behavioral flexibility (EDFLIX) were moderately to strongly associated with greater impairment on occupational, social leisure and interpersonal function as indexed by WSAS scores $\left(r_{\mathrm{s}}=-0.55\right.$ to -0.61 , $p<0.01)$. Higher scores for cognitive-behavioral flexibility showed weak positive associations with dichotomous occupational and social milestones; greater flexibility was associated with attainment of the milestone (working, being in a long-term relationship) $\left(r_{\mathrm{s}}=0.24<0.05\right)$. Higher scores for cognitive-behavioral flexibility showed a weak to moderate association with higher ratings for quality of close interpersonal relationships $\left(r_{\mathrm{s}}=0.37, p<0.01\right)$, but was only weakly associated with greater social contact with friends $\left(r_{\mathrm{s}}=0.21\right.$, $p<0.05)$.

\section{Regression analysis}

Results of the regression analyses conducted to examine whether EDFLIX scores remained significantly associated with functional outcomes beyond known covariates are displayed in Tables 3, 4 and 5. A hierarchical linear regression with age, DASS scores, EDE-Q scores and BMI entered into the model at block 1 and EDFLIX scores entered at block 2 was conducted for each of the WSAS areas of function, and each of the social function composite scores. Beyond the covariates, lower EDFLIX scores were a significant independent predictor of greater WSAS work impairment (accounting for $17.5 \%$ of total variance explained), and greater WSAS social impairment (accounting for $23 \%$ of total variance explained) but were not a significant independent predictor of WSAS relationship impairment. EDFLIX scores were not a significant independent predictor of either of the social function composite scores beyond the covariates. An interaction term (EDFLIX x diagnosis) entered at block 3 confirmed there was no difference in the predictive strength of flexibility scores between participants with a current versus a former diagnosis.

A binary logistic regression with age, DASS scores, EDE-Q scores and BMI entered into the model at block 1 and EDFLIX scores entered at block 2 was conducted for each of the dichotomous functional milestones. Beyond the covariates, higher flexibility scores were a significant independent predictor of attainment of the relationship milestone (being in a long-term relationship, current or former), but not the work milestone (currently working or studying with adequate progression). An interaction term entered at block 3 confirmed there was no difference in the predictive 
Table 3 EDFLIX scores as a predictor of WSAS scores in second block of hierarchical multiple regression

\begin{tabular}{|c|c|c|c|c|c|c|c|c|c|c|}
\hline \multirow[t]{3}{*}{ Block 1} & \multirow[b]{3}{*}{$\begin{array}{l}\text { Age, DASS, } \\
\text { EDE-Q, BMI }\end{array}$} & \multicolumn{3}{|l|}{ WSAS work } & \multicolumn{3}{|l|}{ WSAS social leisure } & \multicolumn{3}{|c|}{ WSAS relationship } \\
\hline & & \multicolumn{3}{|l|}{$\boldsymbol{R}^{2}=0.47 * * *$} & \multicolumn{3}{|l|}{$\boldsymbol{R}^{2}=0.42 * * *$} & \multicolumn{3}{|l|}{$R^{2}=0.42 * * *$} \\
\hline & & $b$ & $\mathrm{SE}_{b}$ & $\beta$ & $b$ & $\mathrm{SE}_{b}$ & $\beta$ & $b$ & $\mathrm{SE}_{b}$ & $\beta$ \\
\hline \multirow[t]{8}{*}{ Block 2} & Age & 0.03 & 0.02 & 0.09 & $<0.01$ & 0.02 & $<0.01$ & $<0.01$ & & \\
\hline & Depression & 0.06 & 0.02 & $0.31 *$ & 0.03 & 0.02 & 0.17 & 0.04 & 0.03 & 0.18 \\
\hline & Anxiety & 0.04 & 0.03 & 0.17 & 0.01 & 0.03 & 0.05 & 0.02 & 0.03 & 0.07 \\
\hline & Stress & $<0.01$ & 0.04 & $<0.01$ & 0.01 & 0.04 & 0.05 & 0.05 & 0.04 & 0.21 \\
\hline & EDE-Q & -0.01 & 0.21 & $<0.01$ & 0.11 & 0.22 & 0.07 & 0.10 & 0.23 & 0.07 \\
\hline & BMI & -0.09 & 0.06 & -0.13 & -0.10 & 0.06 & -0.15 & -0.06 & 0.06 & -0.09 \\
\hline & EDFLIX & -0.02 & 0.01 & $-0.30 *$ & -0.03 & 0.01 & $-0.40 * *$ & -0.02 & 0.01 & -0.22 \\
\hline & & \multicolumn{3}{|l|}{$R^{2}$ change $=.03 *$} & \multicolumn{3}{|l|}{$R^{2}$ change $=.05^{* *}$} & \multicolumn{3}{|l|}{$R^{2}$ change $=.02$} \\
\hline
\end{tabular}

Significant values in bold (including marginal)

$*=p<0.05, * *=p<0.01, * * *=p<0.001 ; b=$ unstandardized regression coefficient; $\beta=$ standardized regression coefficient. All predictor variables are mean-centered

$B M I$ Body Mass Index, DASS Depression Anxiety Stress Scale short form, EDE- $Q$ Eating Disorder Examination Questionnaire, EDFLIX Eating Disorder Flexibility Index, WSAS Work and Social Adjustment Scale

Table 4 EDFLIX scores as a predictor of dichotomous milestones in second block of logistic regression

\begin{tabular}{|c|c|c|c|c|c|c|c|c|c|}
\hline & \multicolumn{3}{|c|}{ Occupational milestone } & \multirow[t]{2}{*}{$95 \% \mathrm{CI}$} & \multicolumn{3}{|c|}{ Relationship milestone } & \multirow[b]{2}{*}{$95 \% \mathrm{CI}$} \\
\hline & & $B$ & SE & $\operatorname{Exp}(b)$ & & $b$ & SE & $\operatorname{Exp}(b)$ & \\
\hline \multirow[t]{7}{*}{ Block 2} & Age & -0.01 & 0.3 & 0.99 & $0.89-1.03$ & 0.06 & $\mathbf{0 . 0 3}$ & $1.06^{*}$ & $1.01-1.12$ \\
\hline & Depression & -0.04 & 0.04 & 0.96 & $0.89-1.03$ & -0.02 & 0.03 & 0.98 & $0.93-1.04$ \\
\hline & Anxiety & -0.03 & 0.04 & 0.97 & $0.89-1.06$ & $<0.01$ & 0.03 & 1.01 & $0.94-1.07$ \\
\hline & Stress & 0.04 & 0.06 & 1.04 & $0.94-1.16$ & $<0.01$ & 0.04 & 0.99 & $0.92-1.07$ \\
\hline & EDE-Q & -0.19 & 0.36 & 0.83 & $0.41-1.68$ & 0.29 & 0.24 & 1.33 & $0.83-2.15$ \\
\hline & BMI & -0.02 & 0.08 & 0.98 & $0.84-1.14$ & 0.06 & 0.06 & 1.07 & $0.94-1.20$ \\
\hline & EDFLIX & 0.01 & 0.02 & 1.01 & $0.98-1.05$ & 0.03 & 0.01 & $1.03 *$ & $1.0-1.05$ \\
\hline
\end{tabular}

Significant values in bold (including marginal)

$*=p<.05, * *=p<.01, * * *=p<.001 ; b=$ unstandardized regression coefficient; $\beta=$ standardized regression coefficient. All variables in regression mean-centered

$B M I$ Body Mass Index, EDE- $Q$ Eating Disorder Examination Questionnaire, WSAS Work and Social Adjustment Scale

strength of flexibility scores between participants with a current versus former diagnosis.

\section{Discussion}

The current research measured self-reported cognitivebehavioral flexibility as indexed by the EDFLIX, and objective social and occupational functional milestones to investigate the relationship between flexibility and everyday function in adults with lifetime AN. We also measured subjective social and occupational function using the WSAS to facilitate comparison with other research, and to assess associations between objective and subjective measures.

Overall, flexibility scores were significantly associated with subjective, but not objective work and social impairment. Lower flexibility scores were significantly related to higher ratings for impairment in ability to work as indexed by the WSAS but were not associated with poor occupational outcomes such as being unemployed or not showing adequate academic progression. Work participation in the sample was very high, and most students reported standard progression and distinction level grades. Lower flexibility scores were also significantly related to higher ratings for impairment in ability to engage in social leisure 
Table 5 EDFLIX scores as a predictor of composite social function scores in second block of hierarchical multiple regression

\begin{tabular}{|c|c|c|c|c|c|c|c|}
\hline \multirow[t]{3}{*}{ Block 1} & \multirow[b]{3}{*}{$\begin{array}{l}\text { Age, DASS, } \\
\text { EDE-Q, BMI }\end{array}$} & \multicolumn{3}{|c|}{ Frequency of social contact with friends } & \multicolumn{3}{|c|}{$\begin{array}{l}\text { Ratings for quality of close } \\
\text { relationships }\end{array}$} \\
\hline & & \multicolumn{3}{|c|}{$R^{2}=0.18 * *$} & \multicolumn{3}{|c|}{$\boldsymbol{R}^{2}=0.20 * * *$} \\
\hline & & $b$ & $\mathrm{SE}_{b}$ & $\beta$ & $b$ & $\mathrm{SE}_{b}$ & $\beta$ \\
\hline \multirow[t]{8}{*}{ Block 2} & Age & -0.05 & 0.02 & $-0.26 * *$ & $<0.01$ & $<0.01$ & 0.02 \\
\hline & Depression & -0.03 & 0.02 & -0.23 & -0.02 & $<0.01$ & $-.35 *$ \\
\hline & Anxiety & -0.01 & 0.03 & -0.08 & $<0.01$ & $<0.01$ & $<0.01$ \\
\hline & Stress & 0.01 & 0.03 & 0.07 & $<0.01$ & 0.01 & 0.08 \\
\hline & EDE-Q & 0.11 & 0.18 & 0.11 & -0.05 & 0.07 & -0.13 \\
\hline & BMI & 0.04 & 0.05 & 0.10 & -0.01 & 0.07 & -0.07 \\
\hline & EDFLIX & 0.01 & $<0.01$ & $-0.30 *$ & $<0.01$ & $<0.01$ & 0.11 \\
\hline & & \multicolumn{3}{|c|}{$R^{2}$ change $=0.02$} & \multicolumn{3}{|c|}{$R^{2}$ change $=<0.01$} \\
\hline
\end{tabular}

Significant values in bold (including marginal)

$*=p<0.05, * *=p<0.01, * * *=p<0.001 ; b=$ unstandardized regression coefficient

$\beta=$ standardized regression coefficient. All variables in regression mean-centered

$B M I$ Body Mass Index, EDE-Q Eating Disorder Examination Questionnaire, WSAS Work and Social Adjustment Scale activities on the WSAS but were not associated with a more objective measure of the frequency of social contact with friends. The average rating for impairment on the WSAS social leisure activities question in this sample indicates marked impairment. However, almost half the participants reported meeting up with friends face-to-face weekly or more often, and over $80 \%$ reported meeting friends at least monthly. Notably, the pattern of results was flipped for relationships: Flexibility scores were not significantly related to impairment in ability to form and maintain close relationships as indexed by the WSAS but were marginally significantly associated with attaining the relationship functional milestone. Higher flexibility scores were associated with a higher likelihood of being in a long-term relationship (current or former). This pattern of results was not different for participants with a current versus a past diagnosis. Partially consistent with our predictions, there were differences in flexibility and functional outcomes between the current and past diagnosis groups. Participants with a current diagnosis did report more difficulties with flexibility and were less likely to attain the occupational milestone. However, importantly, participants with a current diagnosis were not less likely to attain the relationship milestone and did not report a lower frequency of social contact with friends, and there were no significant differences in the strength of effect of flexibility on function between groups.

In relation to our other specific research question, milestones across occupational, social and relationship areas of function were not significantly correlated. This suggests that individuals with a current or past diagnosis of AN have areas of strength and weakness across different areas of their everyday function. In contrast, the WSAS ratings for functional impact across work, social leisure and ability to form and maintain close relationships were moderately strongly correlated, suggesting broad impairment. Overall, the data from the milestones approach presents a more diverse profile in comparison to that provided by the WSAS.

In summary, self-reported cognitive-behavioral flexibility was associated with different areas of function however the pattern of association was not consistent between objective and subjective function measures. Contrary to our prediction, results from the functional milestones suggest that flexibility is generally not a strong determinant of everyday function but may be a factor in maintaining close interpersonal relationships. The pattern of results provided by the WSAS ratings for the perception of impairment were essentially the opposite-lower flexibility scores were significantly related to perceived impairment to the ability to work and engage in social leisure activities, but not the ability to form and maintain close relationships. Therefore, the current WSAS results are consistent with recently reported results demonstrating improved cognitive flexibility as measured by the Brixton Test is significantly associated with lower ratings for disorder-related impairment on the WSAS [12]. However, the inconsistency between the WSAS and milestone results raise questions about the measurement and interpretation of functional outcomes in AN.

The primary conclusion we draw from these results is that to obtain a more balanced assessment of everyday functioning, both subjective and objective measures should be considered. Self-report measures such as the WSAS 
provide important data regarding perceptions of disorderrelated functional impairment. The WSAS is brief, easy to administer, and has been rated highly for user acceptability [7]. Functional milestones are similarly brief, and their addition could add balance to the measurement of social and occupational functioning. This balance may be important in a number of ways. Milestones such as workplace participation, grade retention and maintaining close relationships require sustained effort, which in turn requires goalrelated focus and motivation. Finding sources of motivation which are related to the quality of life, but not related to the disorder, such as goal-achievements related to work participation or financial independence, has been identified as a possible core therapeutic goal [26]. In clinical practice, positive milestones could be used to identify areas of relative strength, such as workplace participation despite perceptions of impairment in ability to work, which could support a strengths-based approach. Areas of strength could be utilized to buffer the negative effects of the disorder on everyday function [21].

Since milestones require sustained effort over a longer period, a milestone approach may also be particularly useful functional outcome measure for intervention studies which include long-term follow-up, or in other longitudinal research designs. It has been suggested that the evaluation of outcomes of Cognitive Remediation Therapy (CRT) in AN should include measures of real-life functioning, similar to those used in schizophrenia [4]. A milestone approach may be of specific utility to address that suggestion. In the current study, flexibility scores increased as ED-symptoms as measured by the EDE-Q decreased, providing further evidence of increased cognitive-behavioral flexibility as a recovery factor, and the utility of the EDFLIX. Although significantly higher, flexibility scores for participants with a past diagnosis were still within the clinical range, therefore the current results suggest the continued utility of CRT in AN after weight recovery has occurred.

Balance is also important in the dissemination of research at a societal level. Although data which note perceptions of severe impairment to work and social function are important to understanding the experience of the impact of the disorder, they may be subject to misinterpretation. On their own, reports that individuals with AN are severely impaired in their ability to work may sound like people are unemployed. Providing additional data which highlights objective strengths such as high workplace participation may help to de-stigmatize living with an eating disorder and promote inclusivity [43]. Increased recognition of high workplace participation in AN may be used to promote more specific investigation of work as a potential recovery factor, and to provide focused support for the everyday challenges posed by the workplace [35].

\section{Strengths and limitations}

To our knowledge, the current study is the first to investigate the relationship between self-reported cognitive-behavioral flexibility and objective functional milestones in $\mathrm{AN}$, and the first to compare subjective and objective functional outcome measures in AN. Strengths include an adequate total sample, analysis by groups which did not differ in age, attainment of the relationship milestone or the social objective outcome measure, and an analytic plan which controlled for the effect of age, mood, BMI and ED-related cognitions on functional outcome. The current research also has limitations which must be considered. Firstly, we measured cognitive-behavioral flexibility using self-report only. As noted in the introduction, self-report and performance tests capture different aspects of the same construct. Therefore, further investigation using performance tests which index both cognitive and behavioral flexibility and objective functional outcomes are recommended. Our sample included adult participants of all ages and stages of recovery from AN, including participants who reported a diagnosis of a partial AN syndrome (EDNOS-AN, OSFED-AN and Atypical AN) which together represent considerable heterogeneity. Although differences by subtype have been identified for cognitive flexibility performance tests, differences in flexibility in everyday life are yet to be evaluated, and where power allows, analysis by AN sub-type, or by symptom dimensions is recommended for future research [44]. Results regarding the impact of cognitive flexibility on functional outcomes would also be strengthened by the use of a control group, however, comparison with healthy controls for the WSAS is difficult without changing the presentation of the items from the validated scale. Finally, comparisons between participants with current and past diagnoses were cross-sectional and causal relationships between flexibility and function cannot be determined.

In conclusion, functional milestones could provide an appropriate outcome measure for future research in AN. Functional milestone data for individuals with a current and past diagnosis of AN presents a more diverse profile than is captured by self-report, demonstrating strengths and weaknesses across different areas of everyday function. Inclusion of objective milestones as a functional outcome measure may be useful in clinical settings to identify personal strengths and in research to provide an outcome measure which indexes sustained effort. We recommend further research in the area of cognitive-behavioral flexibility and everyday function is important in AN to close the gap between the impairment level where we are currently testing, and the participation level where we want to be drawing conclusions [6]. 


\section{What is already known on this subject?}

Cognitive-behavioral flexibility measured using standard neuropsychological performance tests is not strongly associated with self-reported functional outcome in adults with lifetime AN. However, results in adolescent cohorts have demonstrated self-report measures of cognitive-behavioral flexibility appear to be sensitive to the everyday flexibility issues experienced by adolescents with AN.

\section{What this study adds?}

This study is the first to investigate the relationship between flexibility and function in adults with lifetime AN using self-reported cognitive-behavioral flexibility and both subjective and objective functional outcome measures. Flexibility scores were significantly associated with subjective functional outcome, but not objective functional milestones for the same domain. Subjective outcome measures indicate broad impairment, but functional milestones indicate individuals with a lifetime diagnosis of $\mathrm{AN}$ have areas of strength and weakness across their everyday function. Results suggest both subjective and objective function measures should be used in future research.

Supplementary Information The online version contains supplementary material available at https://doi.org/10.1007/s40519-021-01300-7.

Acknowledgements Thank you to all participants who took part in this study

Author contributions All authors have contributed significantly to the manuscript.

Funding This research did not receive any specific grant from funding agencies in the public, commercial or not-for-profit sectors.

Data availability Data is available on request from the first author (KD) and subject to ethics approval.

Code availability Not applicable.

\section{Declarations}

Conflict of interest The authors have no conflict of interest to report. Full author disclosures: PH has prepared a report under contract for (July 2017) and received honoraria for education of psychiatrists from Takeda Pharmaceuticals. ST has received travel grants, research grants and honoraria from Takeda for commissioned reports, public speaking and consultant work. He chairs their Australian Clinical Binge Eating Advisory Board. KD has no disclosures to make.

Ethics approval This study was performed in line with the principles of the Declaration of Helsinki. Approval was granted by The University of Sydney Human Research Ethics Committee, approval number 2020/176.
Informed consent Informed consent was obtained from all participants included the study.

Consent for publication All participants who completed the study indicated as part of their informed consent that they understood the results of the study may be published.

\section{References}

1. Abbate-Daga G, Buzzichelli S, Marzola E, Aloi M, Amianto F, Fassino S (2015) Does depression matter in neuropsychological performances in anorexia nervosa? A descriptive review. Int J Eat Disord 48(6):736-745. https://doi.org/10.1002/eat.22399

2. Arkell J, Robinson P (2008) A pilot case series using qualitative and quantitative methods: biological, psychological and social outcome in severe and enduring eating disorder (anorexia nervosa). Int J Eating Disord 41(7):650-656

3. Bohn K, Doll HA, Cooper Z, O'Connor M, Palmer RL, Fairburn CG (2008) The measurement of impairment due to eating disorder psychopathology. Behav Res Ther 46(10):1105-1110. https://doi. org/10.1016/j.brat.2008.06.012

4. Brockmeyer T, Friederich H-C (2015) Comprehending and fostering cognitive-behavioural flexibility in anorexia nervosa. In: Tchanturia K (ed) Cognitive remediation therapy (CRT) for eating and weight disorders. Routledge, pp 92-108

5. Burgess PW, Shallice T (1997) The hayling and brixton tests, Thames Valley Test Company. Edmonds, England

6. Chan RCK, Shum D, Toulopoulou T, Chen EYH (2008) Assessment of executive functions: review of instruments and identification of critical issues. Arch Clin Neuropsychol 23(2):201-216. https://doi.org/10.1016/j.acn.2007.08.010

7. Crawford MJ, Robotham D, Thana L, Patterson S, Weaver T, Barber R, Rose D (2011) Selecting outcome measures in mental health: the views of service users. J Ment Health 20(4):336-346. https://doi.org/10.3109/09638237.2011.577114

8. Dahlgren CL, Hage TW, Wonderlich JA, Stedal K (2019) General and eating disorder specific flexibility: development and validation of the eating disorder flexibility index (EDFLIX) questionnaire. Front Psychol 10(663). https://doi.org/10.3389/fpsyg.2019.00663

9. Dann KM, Hay P, Touyz S (2021) Are poor set-shifting and central coherence associated with everyday function in anorexia nervosa? A systematic review. J Eat Disord 9(1):40. https://doi.org/ 10.1186/s40337-021-00392-x

10. Depp CA, Mausbach BT, Harmell AL, Savla GN, Bowie CR, Harvey PD, Patterson TL (2012) Meta-analysis of the association between cognitive abilities and everyday functioning in bipolar disorder. Bipolar Disord 14(3):217-226. https://doi.org/10.1111/j. 1399-5618.2012.01011.x

11. Diamond A (2013) Executive functions. Annu Rev Psychol 64(1):135-168. https://doi.org/10.1146/annur ev-psych-113011-143750

12. Duriez P, Kaya Lefèvre H, Di Lodovico L, Viltart O, Gorwood P (2021) Increased cognitive flexibility mediates the improvement of eating disorders symptoms, depressive symptoms and level of daily life functioning in patients with anorexia nervosa treated in specialised centres. Eur Eat Disord Rev 29(4):600-610. https://doi.org/10. 1002/erv. 2829

13. Evans V, Iverson G, Yatham L, Lam R (2014) The relationship between neurocognitive and psychosocial functioning in major depressive disorder. J Clin Psychiatry 75(12):1359-1370. https:// doi.org/10.4088/JCP.13r08939

14. Fairburn CG, Beglin SJ (1994) Assessment of eating disorders: Interview or self-report questionnaire? Int J Eat Disord 16(4):363-370 
15. Faul F, Erdfelder E, Buchner A, Lang A-G (2009) Statistical power analyses using $\mathrm{G}^{*}$ Power 3.1: Tests for correlation and regression analyses. Behav Res Methods 41(4):1149-1160.

16. Grant DA, Berg E (1948) A behavioral analysis of degree of reinforcement and ease of shifting to new responses in a Weigl-type card-sorting problem. J Exp Psychol 38(4):404-411. https://doi. org $/ 10.1037 / \mathrm{h} 0059831$

17. Green MF, Llerena K, Kern RS (2015) The "Right Stuff" revisited: What have we learned about the determinants of daily functioning in schizophrenia? Schizophr Bull 41(4):781-785. https:// doi.org/10.1093/schbul/sbv018

18. Harrison A, Mountford VA, Tchanturia K (2014) Social anhedonia and work and social functioning in the acute and recovered phases of eating disorders. Psychiatry Res 218(1-2):187-194. https://doi. org/10.1016/j.psychres.2014.04.007

19. Harvey PD, Sabbag S, Prestia D, Durand D, Twamley EW, Patterson TL (2012) Functional milestones and clinician ratings of everyday functioning in people with schizophrenia: overlap between milestones and specificity of ratings. J Psychiatric Res 46(12):15461552. https://doi.org/10.1016/j.jpsychires.2012.08.018

20. Henry JD, Crawford JR (2005) The short-form version of the depression anxiety stress scales (DASS-21): construct validity and normative data in a large non-clinical sample. Br J Clin Psychol 44(2):227-239. https://doi.org/10.1348/014466505x29657

21. Holst Y, Thorell LB (2019) Functional impairments among adults with ADHD: a comparison with adults with other psychiatric disorders and links to executive deficits. Appl Neuropsychol 1-13. doi:https://doi.org/10.1080/23279095.2018.1532429

22. Howlett CA, Wewege MA, Berryman C, Oldach A, Jennings E, Moore E, . . Moseley GL (2021). Same room-different windows? A systematic review and meta-analysis of the relationship between self-report and neuropsychological tests of cognitive flexibility in healthy adults. Clinical Psychology Review, 102061. doi: https://doi.org/10.1016/j.cpr.2021.102061

23. Lounes N, Khan G, Tchanturia K (2011) Assessment of cognitive flexibility in anorexia nervosa-Self-report or experimental measure? A brief report. J Int Neuropsychol Soc 17(5):925-928. https://doi.org/10.1017/S1355617711000671

24. Lovibond PF, Lovibond SH (1995) The structure of negative emotional states: comparison of the depression anxiety stress scales (DASS) with the beck depression and anxiety inventories. Behav Res Ther 33(3):335-343

25. McAnarney ER, Zarcone J, Singh P, Michels J, Welsh S, Litteer T, Klein JD (2011) Restrictive anorexia nervosa and set-shifting in adolescents: a biobehavioral interface. J Adolesc Health 49(1):99101. https://doi.org/10.1016/j.jadohealth.2010.11.259

26. Mitchison D, Dawson L, Hand L, Mond J, Hay P (2016) Quality of life as a vulnerability and recovery factor in eating disorders: a community-based study. BMC Psychiatry Vol 16 2016, ArtID 328

27. Miyake A, Friedman NP, Emerson MJ, Witzki AH, Howerter A, Wager TD (2000) The unity and diversity of executive functions and their contributions to complex "Frontal Lobe" tasks: a latent variable analysis. Cogn Psychol 41(1):49-100. https://doi.org/10. 1006/cogp.1999.0734

28. Mond JM, Hay PJ, Rodgers B, Owen C (2006) Eating disorder examination questionnaire (EDE-Q): norms for young adult women. Behav Res Ther 44(1):53-62. https://doi.org/10.1016/j. brat.2004.12.003

29. Mundt JC, Marks IM, Shear MK, Greist JM (2002) The work and social adjustment scale: a simple measure of impairment in functioning. Br J Psychiatry 180(5):461-464

30. Oldershaw A, Lavender T, Schmidt U (2018) Are socio-emotional and neurocognitive functioning predictors of therapeutic outcomes for adults with anorexia nervosa? Euro Eating Disord Rev 26(4):346-359. https://doi.org/10.1002/erv.2602
31. Perna G, Cavedini P, Harvey PD, Di Chiaro NV, Daccò S, Caldirola D (2016) Does neuropsychological performance impact on real-life functional achievements in obsessive-compulsive disorder? A preliminary study. Int J Psychiatry Clin Pract 20(4):224231. https://doi.org/10.1080/13651501.2016.1223856

32. Renwick B, Musiat P, Lose A, DeJong H, Broadbent H, Kenyon M, Schmidt U (2015) Neuro- and social-cognitive clustering highlights distinct profiles in adults with anorexia nervosa. Int $\mathrm{J}$ Eat Disord 48(1):26-34. https://doi.org/10.1002/eat.22366

33. Roberts ME, Barthel FM, Lopez C, Tchanturia K, Treasure JL (2011) Development and validation of the detail and flexibility questionnaire (DFlex) in eating disorders. Eat Behav 12(3):168 174. https://doi.org/10.1016/j.eatbeh.2011.04.001

34. Schmidt U, Treasure J (2006) Anorexia nervosa: Valued and visible. A cognitive-interpersonal maintenance model and its implications for research and practice. Br J Clin Psychol 45(3):343-366. https://doi.org/10.1348/014466505x53902

35. Siegel JA, Sawyer KB (2018) Eating disorders in the workplace: a qualitative investigation of women's experiences. Psychol Women Q 43(1):37-58. https://doi.org/10.1177/0361684318812475

36. Stedal K, Dahlgren CL (2015) Neuropsychological assessment in adolescents with anorexia nervosa-exploring the relationship between self-report and performance-based testing. J Eat Disord 3(1):27. https://doi.org/10.1186/s40337-015-0062-2

37. Strassnig MT, Raykov T, O'Gorman C, Bowie CR, Sabbag S, Durand D, Harvey PD (2015) Determinants of different aspects of everyday outcome in schizophrenia: the roles of negative symptoms, cognition, and functional capacity. Schizophr Res 165(1):76-82. https://doi.org/10.1016/j.schres.2015.03.033

38. Sy R, Ponton K, De Marco P, Pi S, IsHak WW (2013) Quality of life in anorexia nervosa: a review of the literature. Eat Disord 21(3):206-222. https://doi.org/10.1080/10640266.2013.779176

39. Tchanturia K, Hambrook D, Curtis H, Jones T, Lounes N, Fenn K, Davies H (2013) Work and social adjustment in patients with anorexia nervosa. Compr Psychiatry 54(1):41-45. https://doi.org/ 10.1016/j.comppsych.2012.03.014

40. Treasure J, Schmidt U (2013) The cognitive-interpersonal maintenance model of anorexia nervosa revisited: a summary of the evidence for cognitive, socio-emotional and interpersonal predisposing and perpetuating factors. J Eat Disord 1(1):13. https://doi. org/10.1186/2050-2974-1-13

41. van Hoeken D, Hoek HW (2020) Review of the burden of eating disorders: mortality, disability, costs, quality of life, and family burden. Curr Opin Psychiatry 33(6):521

42. van Noort BM, Kraus MKA, Pfeiffer E, Lehmkuhl U, Kappel V (2016) Neuropsychological and behavioural short-term effects of cognitive remediation therapy in adolescent anorexia nervosa: a pilot study. Eur Eat Disord Rev 24(1):69-74. https://doi.org/10. 1002/erv.2383

43. van Rensburg MGJ (2021) Transformative eating disorder research: qualitative research considerations. J Eat Disord $9(1): 1-2$

44. Wildes JE, Marcus MD (2013) Incorporating dimensions into the classification of eating disorders: three models and their implications for research and clinical practice. Int J Eat Disord 46(5):396403. https://doi.org/10.1002/eat.22091

45. Wu M, Brockmeyer T, Hartmann M, Skunde M, Herzog W, Friederich HC (2014) Set-shifting ability across the spectrum of eating disorders and in overweight and obesity: a systematic review and meta-analysis. Psychol Med 44(16):3365-3385. https://doi.org/ $10.1017 /$ S0033291714000294

Publisher's Note Springer Nature remains neutral with regard to jurisdictional claims in published maps and institutional affiliations. 